\title{
Instrument reflections and scene amplitude modulation in a polychromatic microwave quadrature interferometer
}

\author{
Chris C. Dobson, Jonathan E. Jones, and Greg Chavers \\ Propulsion Research Center, NASA / Marshall Space Flight Center, Huntsville, Alabama 35812
}

A polychromatic microwave quadrature interferometer has been characterized using several laboratory plasmas. Reflections between the transmitter and the receiver have been observed, and the effects of including reflection terms in the data reduction equation have been examined. An error analysis which includes the reflections, modulation of the scene beam amplitude by the plasma, and simultaneous measurements at two frequencies has been applied to the empirical database, and the results are summarized. For reflection amplitudes around $10 \%$, the reflection terms were found to reduce the calculated error bars for electron density measurements by about a factor of 2. The impact of amplitude modulation is also quantified. In the complete analysis, the mean error bar for highdensity measurements is $7.5 \%$, and the mean phase shift error for low-density measurements is $1.2^{\circ}$.

PACS no.'s/OCIS codes, etc analogue

\section{Introduction.}

A millimeter wave interferometer which uses a beam consisting of three discrete frequencies has been used to measure phase shifts in laboratory plasmas. This instrument was designed and built as a primary diagnostic tool for plasma experiments in support of research on propulsion technologies. The design was derived from several sources, notably including the UCLA Large Plasma Device $70 \mathrm{GHz}$ quadrature instrument [1], a $35 \mathrm{GHz}$ (heterodyne) interferometer used at Forschungszentrum für Mikrostrukturtechnik [2], and discussion of early microwave interferometer experiments by Heald and Wharton [3]. The microwave frequencies provide good sensitivity, and incorporation of phase shifters and additional detectors provides the benefits of quadrature capability [4]. Generally, interferometry at multiple frequencies is useful in species discrimination [5], multiple fringe identification, and error analysis. In the present instrument, the three frequency components, while spatially overlapped, are essentially independent, and the interferometer may be used for mono- or bi-chromatic measurements without operational modification. Also, although not addressed here, the interferometer has reflectometry capability, which, along with the polychromaticity, is suited to operation near critical density, as in, for example, investigation of collision frequencies in transient plasmas [3].

The scene beam of this instrument is intended for applications in which the transmitter and receiver are separated by a distance of approximately one meter. Depending in part on the alignment, it was observed that reflections set up between the transmitter and receiver resulted in significant effects on the phase shift measurements [3]. Probable candidate causes for this circumstance are thought to be (1) the polychromatic design of the interferometer, in which all three frequencies travel in a common wave guide, (2) the optical configuration, which is essentially symmetric with respect to transmitter and receiver, and (3) the flexibility in alignment due to the use of cables, rather than waveguides, in connecting the transmitter and receiver. The inclusion of these reflections in the data reduction model was found to reduce the errors calculated for the phase shift measurements.

An analysis of data collected with this interferometer is presented here, based largely on the results of electron density measurements made in two experimental devices, a high-density magnetized plasma column and a low-density electrostatic discharge. The high-density plasma is the core of the Variable Specific Impulse Magnetoplasma Rocket (VASIMR) propulsion testbed and is generated in a long-pulse mode (a few sec.) [6]. The low-density device is a spherical Inertial Electrostatic Confinement (IEC) chamber operating in a sustained discharge mode [7]. A total of 3685 phase shift measurements are included in the database, taken from 312 experimental runs in plasmas from three species. The phase shifts measured ranged from $<0.5^{\circ}$ to $530^{\circ}$, corresponding to areal electron densities from $\approx 10^{11} \mathrm{~cm}^{-2}$ to $\approx 5 \times 10^{13} \mathrm{~cm}^{-2}$. These phase shifts were determined with the standard interferometer equation as well as with an equation including reflections, and the results are compared. For the present analysis the VASIMR data are evaluated at two simultaneous frequencies, and the IEC data at two frequencies sequentially. Also, attenuation of the scene beam, or more generally, modulation of its amplitude, during plasma operations is important in many cases. A Monte Carlo error analysis was performed for this data, and statistical distribution functions for the error bars, as well as for the phase shift differences among models, are compiled from the results. 


\section{The Instrument}

The interferometer consists of a transmitter and receiver connected by coaxial cables. The microwaves are generated on the transmitter and reference signals for the three frequencies are transmitted, at reduced frequencies, over the connecting cables to the receiver, where they are multiplied before combination with the scene components. The working frequencies are 70,90 , and $110 \mathrm{GHz}$, and the scene beam spatially superposes these frequencies in a nominally Gaussian transverse profile using Millitech Gaussian focusing and collection optics. The separation of the transmitter and receiver is variable about the nominal 1 meter and the scene beam waist is located approximately $60 \mathrm{~cm}$ from the transmitter. The full-width at half-maximum of the waist is about $1.8 \mathrm{~cm}$ at $70 \mathrm{GHz}$. For each of the three frequencies, the reference and scene components are each split into two signals before combination at the receiver, and one of the two is shifted in phase to produce a quadrature arrangement. A schematic drawing is shown in Fig. 1.

The spatial distribution of microwave irradiance in the scene beam was mapped for the $70 \mathrm{GHz}$ component using an Epsilon Lambda crystal detector. The detector face is a $2.9 \mathrm{~cm}$ diameter metal disk with a $0.27 \times 0.55$ $\mathrm{cm}$ rectangular aperture in its center. The detector was placed in front of the transmitter optic and translated axially and transversely while recording voltages output

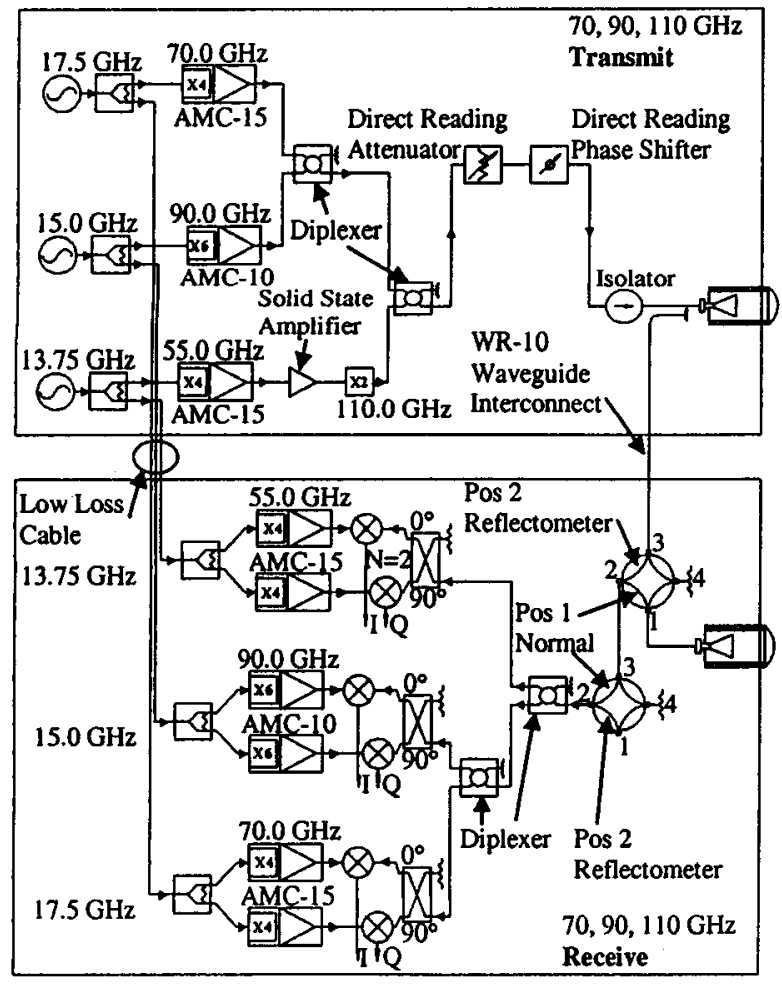

Figure 1 Schematic of the microwave interferometer.

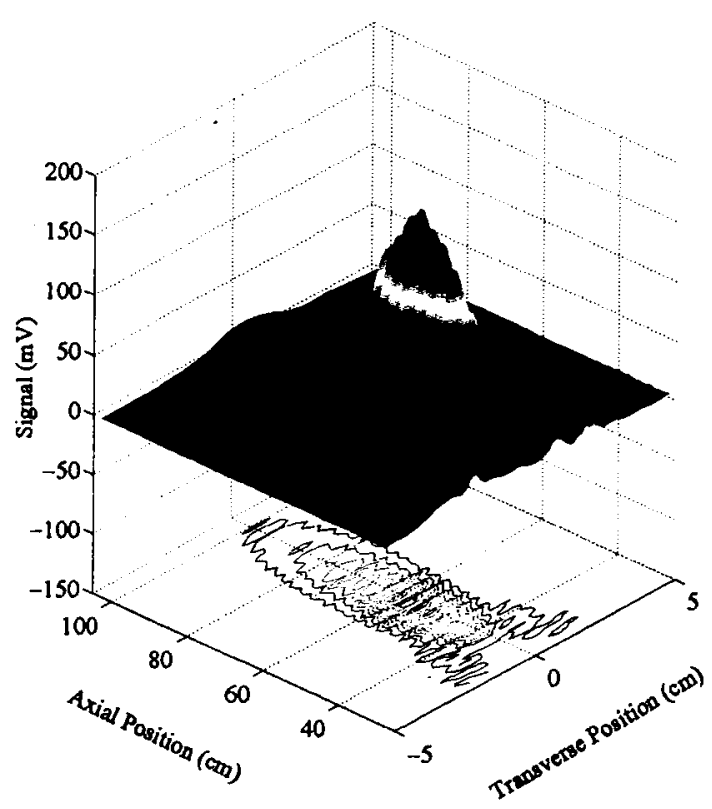

Figure 2 Measured spatial distribution of microwave irradiance for the $70 \mathrm{GHz}$ beam. The measurement grid spacing is $1 \mathrm{~cm}$ axially and $0.16 \mathrm{~cm}$ transversely.

from a preamp attached to the detector. The transverse translation was accomplished using a stepper motor and the axial translation was performed manually. In the mapping, a standing wave pattern was observed between the transmitter and the detector, giving rise to axial oscillations of the measured irradiance of the microwaves at the detector. Fig. 2 gives the results of the mapping. The horizontal axes give the axial and transverse positions, in $\mathrm{cm}$, and the vertical axis gives the signal strength (in $\mathrm{mV}$ ). The axial position is measured from the transmitter optic, and the transverse position from an arbitrary zero point chosen near the transverse maximum. The axial spatial resolution of Fig. 2 is $1 \mathrm{~cm}$ and the oscillation due to the reflection is not resolved, but is evident at an aliased period of $\cong 2.9$ $\mathrm{cm}(\lambda / 2=0.214 \mathrm{~cm})$. Fig. 3a gives a plot of a single transverse profile taken at an axial distance of $60.2 \mathrm{~cm}$, and Fig. $3 \mathrm{~b}$ gives a plot of the axial profile near the waist, scanned with a stepper motor at the central transverse position. The axial oscillations of Fig. 3b have an amplitude approximately $30 \%$ the mean signal.

During normal operation of the interferometer, reflections similar to those observed in the beam mapping, but less marked, were also observed. These reflections occur between the transmitter and receiver, and their strength, and the degree to which they affected the interferometry, varied with the alignment of the instrument and with the experimental configuration. Also, the reflections were usually less apparent in the 

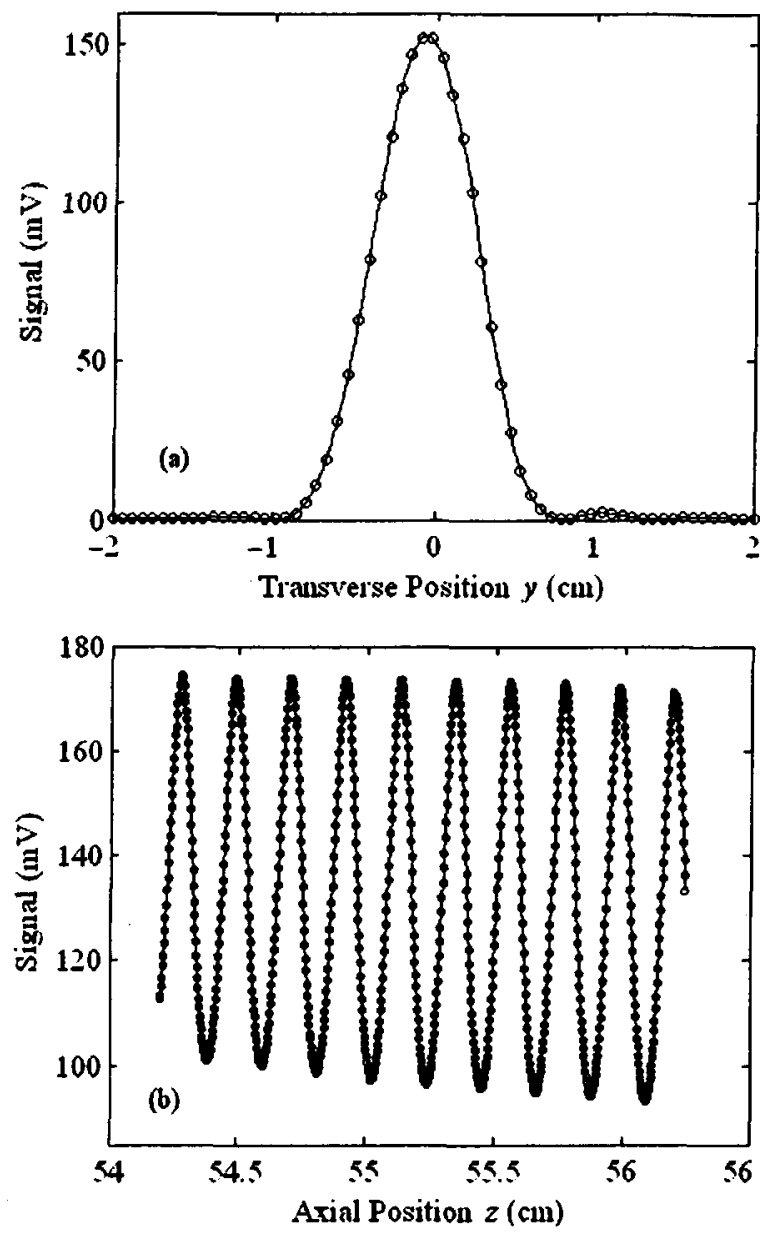

FIG. 3. Measured transverse (a) and axial (b) profiles of the $70 \mathrm{GHz}$ beam. The discrete points are the measurements. The data in (a) are from Fig. 2, at an axial position of 60.2 $\mathrm{cm}$. The oscillations in (b) are due to standing waves set up by the detector.

$90 \mathrm{GHz}$ beam than at the other frequencies. In discussing the reflections, it is important to note the distinction between spatial oscillation and amplitude modulation. The oscillations of Fig. $3 \mathrm{~b}$ are due, like ideal interferometer signals, to the phase relation between waves of constant amplitude. In contrast to this are changes in the amplitude of the collected scene beam signal at the interferometer detector due to, for example, changes in the source output or modified coupling due to the plasma [3].

The interferometer is equipped with a rotary phase shifter which varies the optical path length, but not the physical path length, for the scene beam upstream of the transmitter optics. However, because the plasma itself (or other scene target) varies the phase of the scene beam downstream of the transmitter optics, calibration of the interferometer was performed by translating the transmitter along the optical axis $z$ with a stepper motor, called here the phase motor [1]. In particular, the effect of scene beam reflections, which depends on the optical path length between the transmitter and receiver, is in this way incorporated in the calibration. The translation was made possible because of the flexible connection between transmitter and receiver. The general procedure is to perform a calibration scan just prior to the plasma run. Then the transmitter is stationed at the measurement position $z_{s}$, and data are acquired during the formation and dissolution of the plasma. The calibration scans were usually made over a range from $z_{s}-d$ to $z_{s}+d$, where $d$ is chosen to be slightly larger than half of the maximum wavelength. The rotary phase shifter, while not used for calibration, was used during alignment and it was generally observed that the effect of reflections was not evident in the phase shift pattern produced by this device.

One problem we have encountered in the use of this instrument is failures of the detector diodes. In the course of several tens of hours of operation at three different laboratory facilities, damage to these diodes resulted in the loss of a frequency channel on four occasions. The manufacturer (MilliTech) has suggested that these failures are related to high-voltage pulses which occur episodically and originate in plasma support hardware, such as power supplies. Replacement of the diodes is comparatively straightforward, but in practice it entails a change of the quadrature shift for the affected channel. In addition, there is a degree of variability in the quadrature shift, particularly for the 70 $\mathrm{GHz}$ channel, which is observed in the absence of diode failures. For both of these reasons, the receiver was eventually modified by the addition of adjustable shifters, so that the quadrature may easily be set in general. However, this adjustment was not available for the data presented here, and phase shifts as small as $30^{\circ}$ and as large as $116^{\circ}$ have been used in consequence.

\section{Experimental Plasmas}

This interferometer has been used to make measurements in several experimental plasmas. A database taken from two of those experiments is used for the work here. The first of these is the VASIMR VX-10 device (at JSC in Houston TX), which creates a plasma column by flowing gas down a quartz confinement tube through a helicon antenna. The plasma column is shaped by solenoidal magnetic fields and has a length of approximately 2 meters. A schematic is given in Fig. 4. The data used here were part of a series of experiments on diffusion and recombination in the plasma conducted over a period of four days, and were collected at an axial position at the exit of the confinement tube [6]. The ion-cyclotron resonance heating (ICRH) antenna, normally installed 
just downstream of this position, was removed for these experiments (it is not shown in the figure). The plasma column diameter at this position was approximately 2.4 $\mathrm{cm}$, and the gas was He with typical flow rates around $230 \mathrm{sccm}$. Most of the data were taken at the centerline of the column, but several transverse scans are also included. The VASIMR device was operated in a pulsed mode and the typical duration of a "shot" was approximately 2.3 seconds.

The second experimental device was a spherical IEC chamber, which creates a plasma by static discharge in a neutral gas and confines the plasma using

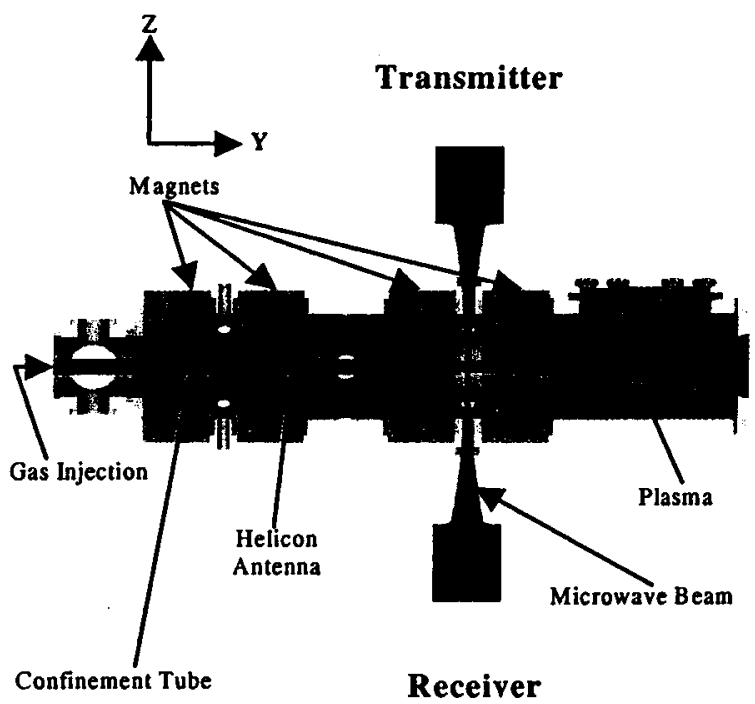

Figure 4 Schematic of VASIMR microwave interferometry.

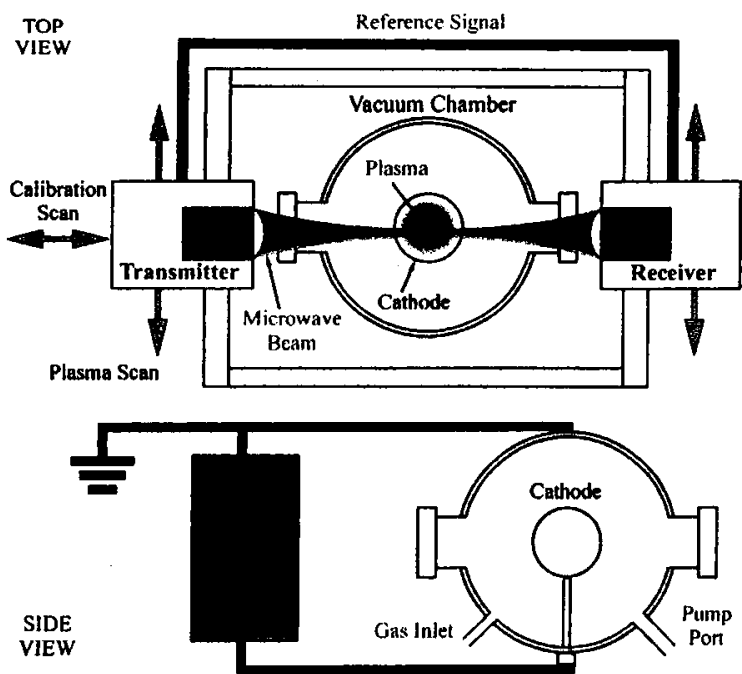

Figure 5 The low density IEC plasma device showing the interferometer calibration and plasma scanning arrangement (top). a radial electrostatic field [7]. A sketch of the IEC device is given in Fig. (5). The vacuum chamber is 60 $\mathrm{cm}$ in diameter, and is jacketed for temperature regulation, producing plasmas which are stable over extended periods of time (typical experiments last many $10^{\prime}$ 's of minutes). It is equipped with two $180^{\circ}$ viewports having clear apertures of $10 \mathrm{~cm}$ diameter. The data used here were collected using two different central cathodes, 15 and $23 \mathrm{~cm}$ in diameter, respectively, over a range of transverse locations within the viewport access. Typical values for the confinement voltages were $\approx 1 \mathrm{kV}$, and for the chamber pressures $\approx 1 \mathrm{~Pa}$. Two gas species, $\mathrm{Ar}$ and $\mathrm{D}_{2}$, are included.

\section{Data Analysis}

In the phase shift analysis here, it is assumed that each of the three frequencies is isolated from the other two. The single frequency which is treated explicitly may thus represent any of the three. For this single frequency, the input channel will be indicated by default, and primes will be used to denote the quadrature channel. Underlining denotes plasma quantities.

The electric field at the microwave detector is a superposition:

$$
E=E_{R} e^{-i \omega t}+\alpha E_{S} e^{i\left(\phi+\phi_{0}-\omega t\right)}\left\lfloor 1+\alpha^{2} \rho e^{i(2 \phi+\beta)}\right\rfloor
$$

where $E$ and $\omega$ are the field amplitude and frequency, respectively; the subscripts $R$ and $S$ denote the reference and scene fields, respectively; $\alpha$ and $\rho$ are the amplitude modulation and reflection coefficients, respectively; $\phi$ is the phase shift of the scene beam relative to its phase at the position $z=0 ; \phi_{0}$ is the phase difference between the reference beam and the non-reflected scene beam at the zero position; and $\beta$ is the phase shift of the reflected component of the scene beam relative to the non-reflected component at the zero position. The amplitude coefficient $\alpha$ is included to account for cases in which there is modulation of the scene beam amplitude when the plasma is present (e.g., refractive bending); by definition $\alpha=1$ when the plasma is absent. Letting $E \rightarrow \sqrt{\gamma c \varepsilon_{0}} E$, where $\gamma$ is the detector responsivity (in volts watt ${ }^{-1} \mathrm{~m}^{2}$ ), $c$ is the speed of light, and $\varepsilon_{0}$ is the permittivity of free space, the voltage output from an interferometer detector is thus modeled as:

$$
\begin{aligned}
& \tilde{V}=E_{R}^{2}+\left(\alpha E_{S}\right)^{2}\left[1+\left(\alpha^{2} \rho\right)^{2}\right]+V_{D}+ \\
& 2 \alpha E_{R} E_{S}\left[\cos \left(\phi+\phi_{0}\right)+\alpha^{2} \rho \cos \left(3 \phi+\phi_{0}+\beta\right)\right] \\
& +2 \alpha^{4} \rho E_{S}^{2} \cos (2 \phi+\beta)
\end{aligned}
$$

where $V_{D}$ is the "dark" voltage. For a quadrature channel, Eq.(2) is unchanged except that the zero phase 
$\phi_{0} \rightarrow \phi_{0}+\phi_{Q} \equiv \phi_{0}^{\prime}$, where $\phi_{Q}$ is the quadrature shift. For $\rho=0$, Eq. (2) reduces to the standard form [1].

Eq. (2) is the basis for the phase shift measurements. The constants, $E_{R}, \phi_{0}$, etc., needed to evaluate the phase in Eq. (2) are denoted $C_{0, k}$, and are treated, for convenience, as the components of a vector $\vec{C}_{0}$, with basis $\hat{c}_{k}, k=1, K$. Other related quantities, identified with the subscript $k$, will also be treated here as vector components in this basis. The $C_{0, k}$ are listed in Table 1 for the three cases used in this work; $K$ is a scale factor included in the phase term for the calibration, $\phi=\kappa z$. The separate reflections case, in which the input and quadrature channels are treated independently, is not physically distinct from the coupled case, but is computationally faster. The theoretical voltage $\tilde{V}$, then, is a function of $\vec{C}_{0}$ and the phase $\phi$; for the calibration data, $\tilde{V}$ is treated as a function of the phase motor position $z$,

$$
\tilde{V}=\tilde{V}\left(z ; \vec{C}_{0}\right)
$$

and for the plasma data, as a function of $\phi=\kappa z_{S}+\Delta \phi$

$$
\tilde{V}=\tilde{V}\left(\phi ; \vec{C}_{0}\right)
$$

where $\Delta \phi$ is the plasma phase shift

The calibration constants $\vec{C}_{0}, \vec{C}_{0}^{\prime}$ were determined from the calibration scans by least squares fitting of the scan data to the model. Specifically, the amplitude residues $R, R^{\prime}$ for the fitting are defined as

$$
\begin{aligned}
R & =\sum_{n=1}^{N}\left[V_{n}-\tilde{V}\left(z_{n} ; \vec{C}_{0}\right)\right]^{2} \\
R^{\prime} & =\sum_{n=1}^{N}\left[V_{n}^{\prime}-\tilde{V}\left(z_{n} ; \vec{C}_{0}^{\prime}\right)\right]^{2}
\end{aligned}
$$

where $V_{n}, V_{n}^{\prime}$ are the microwave signal voltages measured at the phase motor positions $z_{n}$ for $n=1, N$, and the $\vec{C}_{0}, \vec{C}_{0}^{\prime}$ are chosen to minimize $R, R^{\prime}$. Examples of calibration scans and the curve fits based on Eq. (4) are given in Fig. 6 for each of the three frequencies. These examples were chosen specifically to illustrate the instrument reflections. The reflection coefficient $\rho$ is defined as the ratio of the electric field amplitude, incident at the detector, of the reflected component to that of the unreflected component, and the average value for the cases shown in Fig. 6 is $\rho=8.6 \%$. The fractional RMS residue $R_{0} \equiv(R / N)^{1 / 2} /(\Delta V / 2)$, where $\Delta V$ is the peak-to-peak voltage of the sinusoidal oscillation of the calibration scan, has average values of $6.2 \%$ for the standard model and $1.3 \%$ for the reflection model. Again, the deformations caused by $\rho>0$ were not observed in phase scans performed with the upstream rotary shifter.

For the plasma measurements, the scene phase is determined by minimization of the local residues

$r+r^{\prime}=\left[V_{s}-\tilde{V}\left(\phi ; \vec{C}_{0}\right)\right]^{2}+\left[V_{s}^{\prime}-\tilde{V}\left(\phi ; \vec{C}_{0}^{\prime}\right)\right]^{2}$.

In this case, the constants $\vec{C}_{0}, \vec{C}_{0}^{\prime}$ are fixed to their calibration values and the minimization is accomplished either by (1) the variation of $\phi$ alone, or (2) by simultaneous variation of $\phi$ and $\alpha$. The plasma phase shift is then given by the difference, $\Delta \phi=\underline{\phi}-\phi$, (6) for paired voltages with the plasma $\left(\underline{V}_{S}, \underline{V}_{S}^{\prime}\right)$ and without the plasma $\left(V_{S}, V_{S}^{\prime}\right)$. For the case $\rho=0$, an analytic solution $\phi(\tilde{V})$ exists [1].

Monte Carlo simulation was used to estimate the uncertainty in the phase shift measurements. The contributing error sources were taken to be uncertainties in (1) the calibration constants, and (2) the plasma measurement voltages. The calibration constant uncertainties represent systematic errors, "fossilized" from the calibration data [9]. Each of the fitted calibration constants $C_{0, k}$ was taken to be the mean of Gaussian distribution $f\left(C_{k}\right)$ with standard deviation $\sigma_{k}$. A theoretical residue $\widetilde{R}$ was defined,

$$
\begin{aligned}
& \tilde{R}(\vec{C}) \equiv \sum_{n=1}^{N}\left[\tilde{V}\left(z_{n} ; \vec{C}_{0}\right)-\tilde{V}\left(z_{n} ; \vec{C}\right)\right]^{2} \\
& \cong \sum_{n=1}^{N} \sum_{k=1}^{K}\left[\frac{\partial \tilde{V}}{\partial C_{k}}\left(C_{k}-C_{0, k}\right)\right]^{2},
\end{aligned}
$$

and the $\sigma_{k}$ were determined from the conditions that (1) the mean value of $\tilde{R}$ be equal to the measured

Table 1 Calibration constants for fitting of calibration scans to the model, Eq. (2), for 3 cases.

\begin{tabular}{|c|c|c|c|}
\hline Reflections & $\begin{array}{c}\text { No. of } \\
\text { parameters }\end{array}$ & $C_{0, k}$ & $C_{0, k}^{\prime}$ \\
\hline None & $2 \times 4$ & $E_{R}^{2}+E_{S}^{2}+V_{D}, 2 E_{R} E_{S}, \phi_{0}, \kappa$ & $E_{R}^{\prime 2}+E_{S}^{\prime 2}+V_{D}^{\prime}, 2 E_{R}^{\prime} E_{S}^{\prime}, \phi_{0}^{\prime}, \kappa^{\prime}$ \\
\hline Separate & $2 \times 7$ & $E_{R}, E_{S}, \phi_{0}, \kappa, V_{D}, \rho, \beta$ & $E_{R}^{\prime}, E_{S}^{\prime}, \phi_{0}^{\prime}, \kappa^{\prime}, V_{D}^{\prime}, \rho^{\prime}, \beta^{\prime}$ \\
\hline Coupled & 11 & $E_{R}, E_{S}, \phi_{0}, \kappa, V_{D}, \rho, \beta$ & $E_{R}^{\prime}, E_{S}^{\prime}, \phi_{0}^{\prime}-\phi_{0}, \kappa, V_{D}^{\prime}, \rho, \beta$ \\
\hline
\end{tabular}



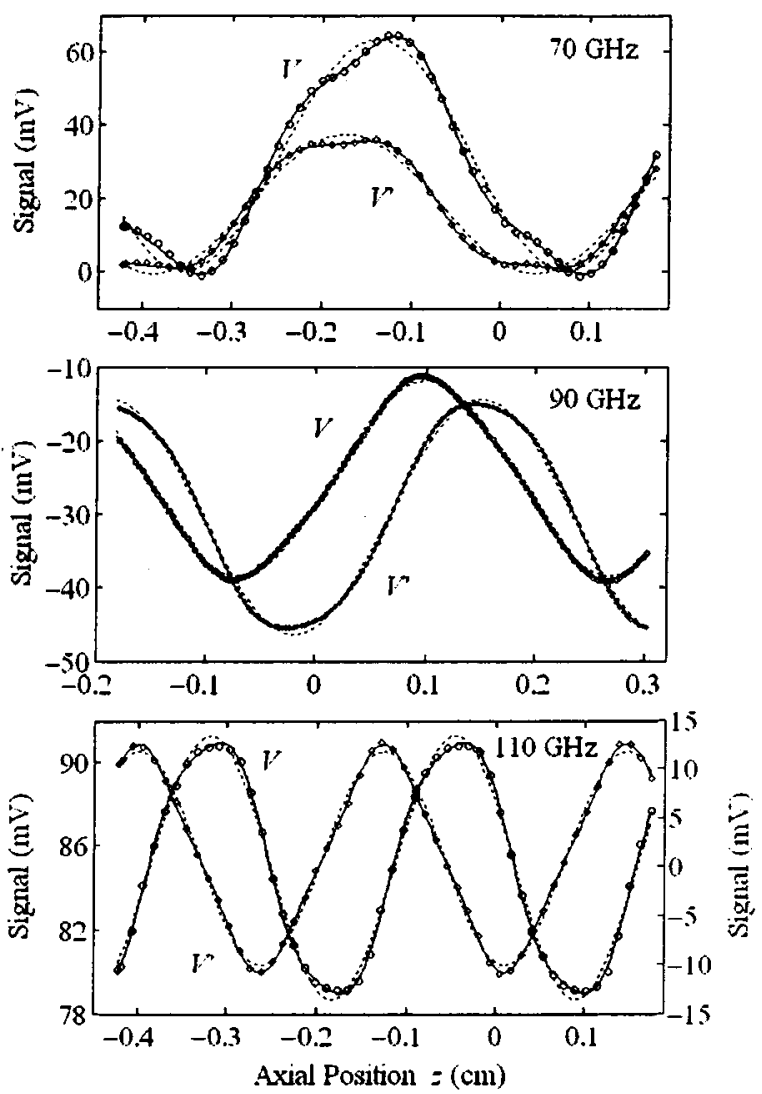

Figure 6 Calibration scans for the interferometer. The circles are the input channel voltages $V$ and the diamonds are the quadrature channel $V^{\prime}$. The broken lines are fits to the standard model and the solid lines are fits to the reflection model. The reflection coefficients $\rho$, and fractional RMS fit residues $R_{0}, R_{0}^{\prime}$ for the standard and reflection cases respectively, are:

$70 \mathrm{GHz}: \rho=12.6 \%, R_{0}=8.5,1.7 \%, R_{0}^{\prime}=9.7,1.9 \%$;

$90 \mathrm{GHz}: \rho=\rho^{\prime}=5.6 \%, R_{0}=3.7,0.71 \%, R_{0}^{\prime}=4.2,0.66 \%$;

$110 \mathrm{GHz}: \rho=7.7 \%, R_{0}=6.0,1.7 \%, R_{0}^{\prime}=5.1,1.4 \%$.

residue,

$$
\overline{\tilde{R}(\vec{C})} \cong \sum_{k=1}^{K} \sigma_{k}^{2} \sum_{n=1}^{N}\left(\frac{\partial \tilde{V}}{\partial C_{k}}\right)^{2}=R
$$

and (2), the individual errors be proportional, statistically, to $\left(\partial \tilde{V} / \partial C_{k}\right)^{-1}$,

$$
\begin{aligned}
& \sigma_{k}^{2} \sum_{n=1}^{N}\left(\frac{\partial \tilde{V}}{\partial C_{k}}\right)^{2} \cong \\
& \sum_{n=1}^{N}\left[\tilde{V}\left(z_{n} ; \vec{C}_{0}\right)-\tilde{V}\left(z_{n} ; \vec{C}_{0}+\hat{c}_{k} \sigma_{k}\right)\right]^{2}=\frac{R}{K}
\end{aligned}
$$

For the measurement voltage, the standard deviation $\sigma_{V}$ of the distribution $f(V)$ about $V_{S}$ was estimated using both the scatter of steady state data and the local residues:

$$
\sigma_{v}=\sqrt{v_{\mathrm{rms}}^{2}+r},
$$

where $v_{\text {rms }}$ is an RMS value of $V_{S}(t)$, taken directly from measurement data for the IEC case and estimated from pre/post test data for the VASIMR case (see Sec. V). Distributions were then calculated from

$$
\begin{aligned}
C_{k . i} & \equiv C_{0, k} \pm \sqrt{2} y_{i} \sigma_{k} \\
V_{i} & \equiv V_{S} \pm \sqrt{2} y_{i} \sigma_{V}
\end{aligned}
$$

where the $y_{i}$ are derived from random numbers $x_{i}$ between 0 and 1 such that $\operatorname{erf}\left(y_{i}\right) \equiv \frac{2}{\sqrt{\pi}} \int_{0}^{y_{i}} e^{-t^{2}} d t=x_{i}$, and the index $i$ is for the statistical sampling. From these distributions, distributions for the phases $\phi$ and $\underline{\phi}$ were calculated and fit to Gaussian curves. The error bar for the phase shift measurement is then given by the root-sum-square (RSS) of the fitted widths,

$$
\delta \Delta \phi=\sqrt{\delta \phi^{2}+\delta \phi^{2}} \text {. }
$$

The electron density is related to the phase shift by

$$
\Delta \phi=-k_{\lambda} \int_{\text {path }}\left[1-\left(1-\frac{n(z)}{n_{\lambda}}\right)^{1 / 2}\right] d z \text {, }
$$

where $k_{\lambda}=2 \pi / \lambda$ is the wave number, $n(z)$ is the density and $n_{\lambda}$ is the critical density, at which the plasma becomes opaque [8]. For $n(z) \ll n_{\lambda}$, the phase shift is proportional to the line integral of the density and does not depend on the path length or the shape of the density distribution along the path. Eq. (13) derives from the refractive index of the plasma and assumes $v_{c} \ll \omega$, where $v_{c}$ is the effective collision frequency, which is expected to be the case for the data here (e.g., VASIMR $p \approx 0.1 \mathrm{~Pa}, T_{e} \approx 5 \mathrm{eV}$ ). Also, the microwave polarization for the VASIMR measurements was parallel to the axial magnetic field, so that, in the cold plasma model (thermal velocity $v_{t h} \ll v_{\phi} \approx c$ ), the dispersion is not affected by this field $(\cong 0.4 \mathrm{~T}$ at the measurement position, giving $\omega_{B}^{2} / \omega^{2}=0.026$ at 70 $\mathrm{GHz}$, where $\omega_{B}$ is the gyrofrequency) [3].

Eq. (13), along with the phase shift errors, Eq. (12), then, gives electron densities and density errors for each of the frequency channels of the interferometer. For the analysis here, two simultaneous frequencies are used for the density measurements. For a single data point, the two frequency channels give error distributions $f_{1}, f_{2}$, with means $\mu_{1}, \mu_{2}$ and widths 
$S_{1}, S_{2}$, which may be combined as a scalar product $f_{1} f_{2}$ to obtain a reduced distribution with mean

$$
\mu_{12}=\left(\frac{\mu_{1}}{S_{1}^{2}}+\frac{\mu_{2}}{S_{2}^{2}}\right)\left(S_{1}^{-2}+S_{2}^{-2}\right)^{-1}
$$

Formally, this may be motivated by interpretation of the densities in the individual distributions as paired measurements, one from each frequency channel. If the two distributions have the same mean and width, for example, the reduced distribution has also the same mean and a width reduced by $\sqrt{2}$; that is, the reduced width scales as a standard deviation of means for $N=2$ [9]. For the general case, in which $\mu_{1} \neq \mu_{2}$ and $S_{1} \neq S_{2}$, a local bias error $\beta_{i}$ is defined such that $\beta_{1}+\beta_{2}=\left|\mu_{1}-\mu_{12}\right|+\left|\mu_{2}-\mu_{12}\right|$, subject to $0 \leq \beta_{i} \propto S_{i}$, and the widths of the individual distributions become

$\sigma_{i}^{2}=S_{i}^{2}+\beta_{i}^{2}, \quad i=1,2$,

giving the reduced deviation $\sigma_{0}$,

$\sigma_{0}^{-2}=\sigma_{1}^{-2}+\sigma_{2}^{-2}$,

and mean

$\mu_{0}=\mu_{12}$.

Thus this correction $\beta_{i}$ accounts for any discrepancy in the means $\mu_{1}, \mu_{2}$ while preserving $\mu_{12}$, obtained directly from the original distributions. The reduced distribution is then integral-normalized,

$\int f_{1}\left(\mu_{1}, \sigma_{1}, x\right) f_{2}\left(\mu_{2}, \sigma_{2}, x\right) d x=e^{-r / 2}$,

where

$\gamma \equiv\left(\frac{\mu_{1}}{\sigma_{1}}\right)^{2}+\left(\frac{\mu_{2}}{\sigma_{2}}\right)^{2}-\left(\frac{\mu_{0}}{\sigma_{0}}\right)^{2}$

vanishes for $\mu_{1}=\mu_{2}$. The integral, Eq. (17), which essentially gives the overlap of the two distributions, is used below in comparing the final results among the models.

In the following section, these Gaussian distributions $f$, associated with individual measurements, will be termed populations, to distinguish them from the distributions of population widths to be discussed.

\section{Results and Discussion}

The VASIMR database represents essentially all the helium shots performed during the test series at the confinement tube exit position. It is comprised of 102 separate shots, including a total of 3475 individual phase shift measurements. The measurement sample rate was approximately $13 \mathrm{~Hz}$ for most of the data, but higher rates, up to $\cong 23 \mathrm{~Hz}$, were used in some cases.
For the analysis here, the intent was to provide a representative statistical sample, and only coarse screening of the data for anomalies was performed. Ideally, a calibration scan would be performed for every plasma shot. In the current system, however, constraints on the communications speed for recording the position of the transmitter along the optical axis limited the rate of calibrations, so that the ratio of calibration files to data files for this data is $\cong 0.6$. These constraints also limited the resolution for the calibration scans; a typical resolution was $0.012 \mathrm{~cm}$, corresponding to $10^{\circ}$ at 70 GHz.

Post-test, the calibration scans were fitted to the model, Eq. (2), using both the standard case and the coupled reflections case (Table 1) in Eq. (4). Each shot file was then reduced using the calibration constants from both models at each data point in the file, and this reduction was performed twice, once with fixed $\alpha=1$ and once with $\alpha$ determined from the best fit to Eq. (5). A Monte Carlo simulation using 5000 samples was then performed for each of the 4 results at a point, giving a set of 4 error bars at each point. The error bar is defined here as the unit width (half-width at $e^{-1}$ maximum $=$ $\sqrt{2} \sigma$ ) of the Gaussian population of phase shifts.

The reduced phase shift data were then used to compile distribution functions. All the data from all the shots were pooled together and parsed into intervals, or bins, based on the value of the phase shift or on the value of the amplitude modulation coefficient. The modulated-amplitude reflection model is taken as the reference case, and values from this model are used for the intervals, for both $\Delta \phi$ and $\alpha$. Then the mean value of the error bar in each interval was computed. Also, the mean phase shift differences between each of the models and the reference case were computed for each interval. Generally, the change in amplitude, as well as the magnitude of reflections, is larger for the low frequency channel. For reference, mean and extreme values for the reflection and amplitude coefficients in this database are given in Table 2 (the value $\alpha=0.05$ is an arbitrary minimum set in the data reduction, and occurred identically only a few times). The mean quadrature shifts for the two frequencies were approximately 31 and $116^{\circ}$, respectively, for the 70 and $110 \mathrm{GHz}$ data.

Table 2 Mean and extreme values for the reflection and amplitude coefficients for the VASIMR data.

\begin{tabular}{|c|c|c|c|c|}
\hline \multirow{2}{*}{} & \multicolumn{2}{|c|}{$\rho$} & \multicolumn{2}{c|}{$\alpha$} \\
\cline { 2 - 5 } & $70 \mathrm{GHz}$ & $110 \mathrm{GHz}$ & $70 \mathrm{GHz}$ & $110 \mathrm{GHz}$ \\
\hline Min & 0.103 & 0.0577 & 0.05 & 0.54 \\
\hline Max & 0.191 & 0.110 & 1.27 & 1.28 \\
\hline Mean & 0.131 & 0.0782 & 0.498 & 0.934 \\
\hline
\end{tabular}


The distributions of the error bars over phase shift are shown in Fig. 7. The means for the $70 \mathrm{GHz}$ data are 14.2 and $14.7^{\circ}$, respectively, for fixed and modulated amplitude in the standard model, and 9.3 and $6.8^{\circ}$, respectively, in the reflection model. For the $110 \mathrm{GHz}$ channel, the corresponding numbers are 8.1, 6.5, 5.3 and $3.0^{\circ}$, respectively. The average number of measurements per bin is about 54. Fig. 8 shows the distributions of phase shift differences. The differences show some phase dependence which is common to both frequencies; for example, they are comparatively small around $-130^{\circ}$, and comparatively large near $-200^{\circ}$. The large oscillation evident in the $70 \mathrm{GHz}$ data is due in part to the attenuation, which is more substantial at the low frequency (see Table 2). Essentially all the $70 \mathrm{GHz}$ data with $|\Delta \phi|>320^{\circ}$ have $\alpha<0.5$, and omission of this data also substantially reduces the difference extrema for $|\Delta \phi|<320^{\circ}$. The large differences for the $70 \mathrm{GHz}$ data are also due in part to the departure from quadrature. The error bar distributions are fairly uniform
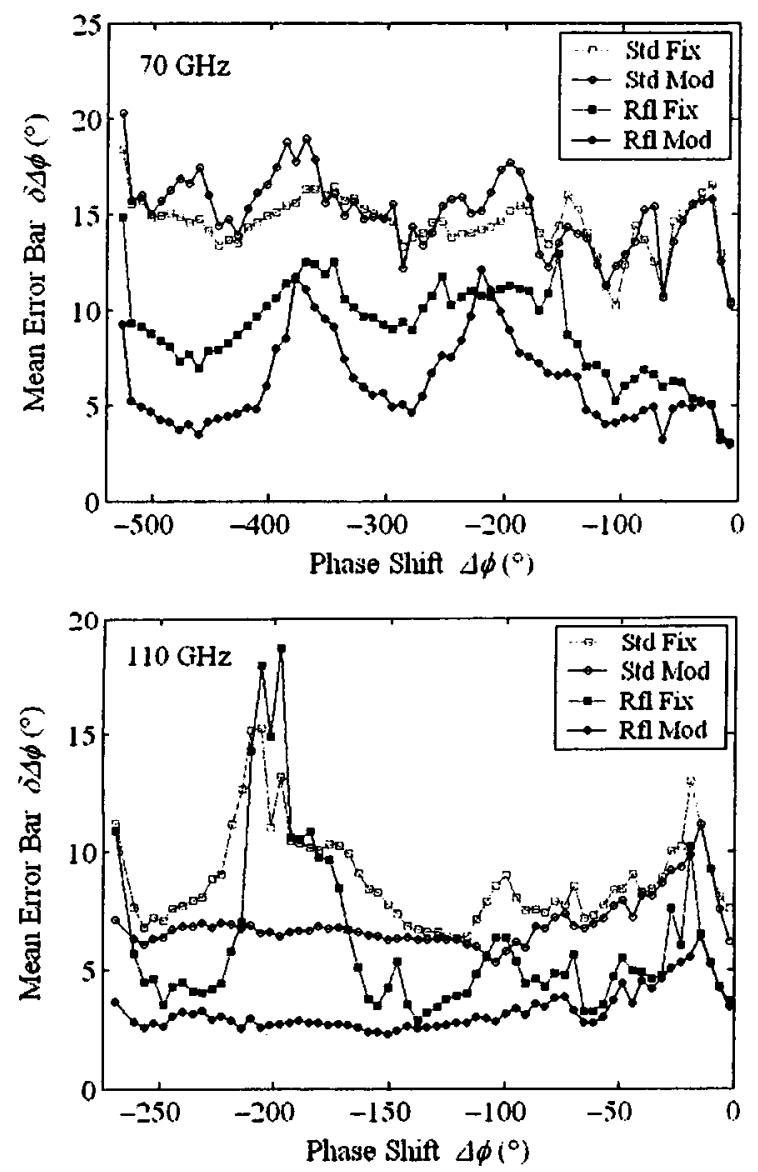

Figure 7 Distribution of the phase shift error bars for the VASIMR data. The squares are fixed amplitude, the circles modulated amplitude. Open symbols are the standard model, solid symbols include reflection. The error bar values are unit widths (half-width at 1/e maximum). between frequencies, and show a mild phase dependence qualitatively similar to that of the differences. However, high frequency maxima in the error distributions are notably absent in the variable amplitude models. The distributions over amplitude coefficient, not given in the figures, showed no pronounced correlations; there was a weak tendency for the errors to decrease with increasing $\alpha<1$, particularly for the standard models.

For comparison, Table 3 gives the results of the data reduction and error analysis applied to several small sets of synthetic data. A fixed set of phases $\phi_{\text {true }}$, giving shifts from 0 to $281^{\circ}$, was used to generate perturbed phase data $\phi_{\varepsilon}, \phi_{\varepsilon}^{\prime}$ by adding to $\phi_{\text {true }}$ random phase errors from a Gaussian population with a unit width of $\delta \phi_{\text {true }}=5^{\circ}$. From the $\phi_{\varepsilon}, \phi_{\varepsilon}^{\prime}$ synthetic voltages $V, V^{\prime}$ were calculated for 8 shot files of 84 points each, using four different quadrature shifts for the two cases $\alpha=1$ and $\alpha=0.66$. Calibration files were

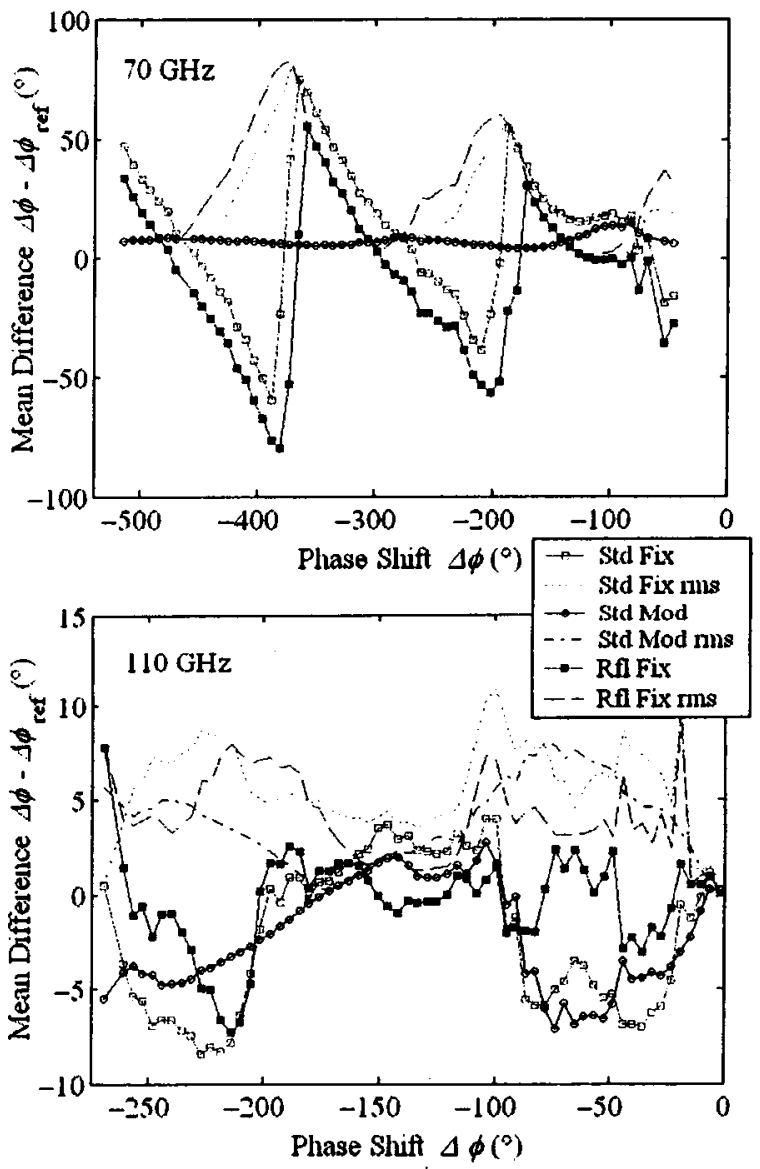

Figure 8 Distribution of the phase shift differences, relative to the reference model, for the VASIMR data. The squares are fixed amplitude, the circles modulated amplitude. Open symbols are the standard model, solid symbols include reflection. Lines with symbols are mean differences and lines without symbols are RMS differences. 
Table 3 Mean values, in degrees, of phase discrepancy magnitudes $\left|\phi-\phi_{\text {true }}\right|$, in italics, and phase shift error bars $\delta \Delta \phi$, in bold, for reduction of synthetic data with $\rho_{\text {true }}=0.1$. The true values are $\overline{\left|\phi_{\varepsilon}-\phi_{\text {true }}\right|}=\pi^{-1 / 2} \delta \phi_{\text {true }}=2.82^{\circ}$ and $\delta \Delta \phi_{\text {true }}=$ $2^{1 / 2} \delta \phi_{\text {truc }}=7.07^{\circ}$.

\begin{tabular}{|c|c|c|c|c|c|c|c|c|c|}
\hline \multirow{2}{*}{$\begin{array}{l}\text { Quadrature } \\
\text { angle } \phi_{Q}\end{array}$} & \multirow{2}{*}{$\alpha_{\text {true }}$} & \multicolumn{4}{|c|}{ Standard $(\rho=0)$} & \multicolumn{4}{|c|}{ Reflections $(\rho>0)$} \\
\hline & & \multicolumn{2}{|c|}{ Fixed $\alpha$} & \multicolumn{2}{|c|}{ Modulated $\alpha$} & \multicolumn{2}{|c|}{ Fixed $\alpha$} & \multicolumn{2}{|c|}{ Modulated $\alpha$} \\
\hline \multirow[b]{2}{*}{$30^{\circ}$} & 1.0 & 3.92 & 11.3 & 5.08 & 11.8 & 1.98 & 8.35 & 3.25 & 8.28 \\
\hline & 0.66 & 20.7 & 12.0 & 3.96 & 13.6 & 16.6 & 10.1 & 3.43 & 11.2 \\
\hline \multirow[b]{2}{*}{$57^{\circ}$} & 1.0 & 3.93 & 10.4 & 4.65 & 10.5 & 2.15 & 7.81 & 2.56 & 7.69 \\
\hline & 0.66 & 15.4 & 20.6 & 3.22 & 11.5 & 13.6 & 21.1 & 2.58 & 9.78 \\
\hline \multirow[b]{2}{*}{$90^{\circ}$} & 1.0 & 4.24 & 10.9 & 4.60 & 10.4 & 2.21 & 8.11 & 2.28 & 8.07 \\
\hline & 0.66 & 8.17 & 23.0 & 3.37 & 11.0 & 8.32 & 20.8 & 2.51 & 9.19 \\
\hline \multirow[b]{2}{*}{$120^{\circ}$} & 1.0 & 4.39 & 12.3 & 4.68 & $\overline{12.5}$ & 2.24 & 8.54 & 2.32 & 8.45 \\
\hline & 0.66 & 14.6 & 19.0 & 3.25 & 12.7 & 14.2 & 15.6 & 2.38 & 9.96 \\
\hline
\end{tabular}

synthesized in a similar manner using the same $\delta \phi_{\text {tree }}$. The resulting data were then processed to produce a set of "measured" phases $\phi$, phase shifts $\Delta \phi$, and error bars $\delta \Delta \phi$ for each of the 8 shot files. The mean phase error, as determined by $\left|\phi-\phi_{\text {true }}\right|$, has values of $9.41^{\circ}$ (fixed $-\alpha$ ) and $4.10^{\circ}(\bmod -\alpha$ ) for the standard models and $7.66^{\circ}($ fixed $-\alpha)$ and $2.66^{\circ}(\bmod -\alpha)$ for the reflection models, which compares with the true value of $2.82^{\circ}$. The mean phase shift error bar $\overline{\delta \Delta \phi}$ for the reference case is $9.08^{\circ}$, as compared with the true value $7.07^{\circ}$. Substantial variation in the results due to the departure from quadrature is generally confined to fixed- $\alpha$ reduction in the attenuation cases. In these cases, $\left|\phi-\phi_{\text {true }}\right|$ shows a phase dependence which is similar to that of the difference distributions in Fig. 8; the magnitude of the effect increases with departure from quadrature and is at a minimum for $\phi_{Q}=90^{\circ}$.

In calculating electron densities from the VASIMR data, the objective here was to determine the fractional error bars and differences. The details of the spatial distribution of the plasma along the optical axis will impact the absolute densities for $n$ of order $n_{\lambda}$, but have little effect on the fractional errors. For the present purpose, then, the plasma distribution is modeled as a step function with a width of $2.45 \mathrm{~cm}$. This diameter is the equivalent width for a Gaussian distribution determined from inversion (uncorrected for the beam profile) of transverse scans made with the interferometer, and is consistent with the visual appearance of the plasma. Using this step function in Eqs. (12) and (13) gives for each phase shift measurement a density and a density error for each of the two frequencies, 70 and $110 \mathrm{GHz}$. These densities were normalized to the low-frequency critical density, $n_{\lambda(70 \mathrm{GHz})}=6.1 \times 10^{13} \mathrm{~cm}^{-3}$, and used to compile distribution functions, just as with the phase shifts. The results for the error bars for combination of the two frequencies as per Eq. (16) are shown in Fig. 9. As a check on the error analysis, Fig. 9 also gives the overlap integral Eq. (17) for the each of the three models compared with the reference model, that is, $e^{-\gamma / 2}$ with $f_{1}$ and $f_{2}$ as reduced density error populations for the comparison and reference models, respectively. The mean values of $e^{-\gamma / 2}$ are 0.80 for the fixed amplitude models and 0.95 for the modulated amplitude. In general, the density error bars are reduced by inclusion of reflection and modulation. Table IV gives mean values for the data of Fig. 9, along with mean values for the fractional density differences for the two frequencies, where the fractional difference is defined as $\Delta n \equiv\left(n_{110 \mathrm{GHz}}-n_{70 \mathrm{GHz}}\right)\left[\frac{1}{2}\left(n_{110 \mathrm{GHz}}+n_{70 \mathrm{GHz}}\right)\right]^{-1}$ and is independent of the error analysis. The behavior of $\overline{|\Delta n|}$ for the different models is qualitatively similar to that of the error bar $\delta n$, although the effect of modulation is more pronounced than that of reflection for the differences. $\overline{\Delta n}$ itself indicates the tendency $n_{110 \mathrm{GHz}}<n_{70 \mathrm{GHz}}$.

For the IEC data, a single phase shift measurement was based on data collected during several contiguous plasma cycles, each having a typical duration of approximately 10 seconds. Such measurements were made over a range of neutral background pressures and transverse locations, and separate calibration files were obtained for each measurement. The voltages from the interferometer $\left(\underline{V}_{s}, \underline{V}_{s}^{\prime}\right.$ and $\left.V_{s}, V_{s}^{\prime}\right)$ used for phase determinations were averages taken over steady state windows within the plasma cycles. Given the voltages 

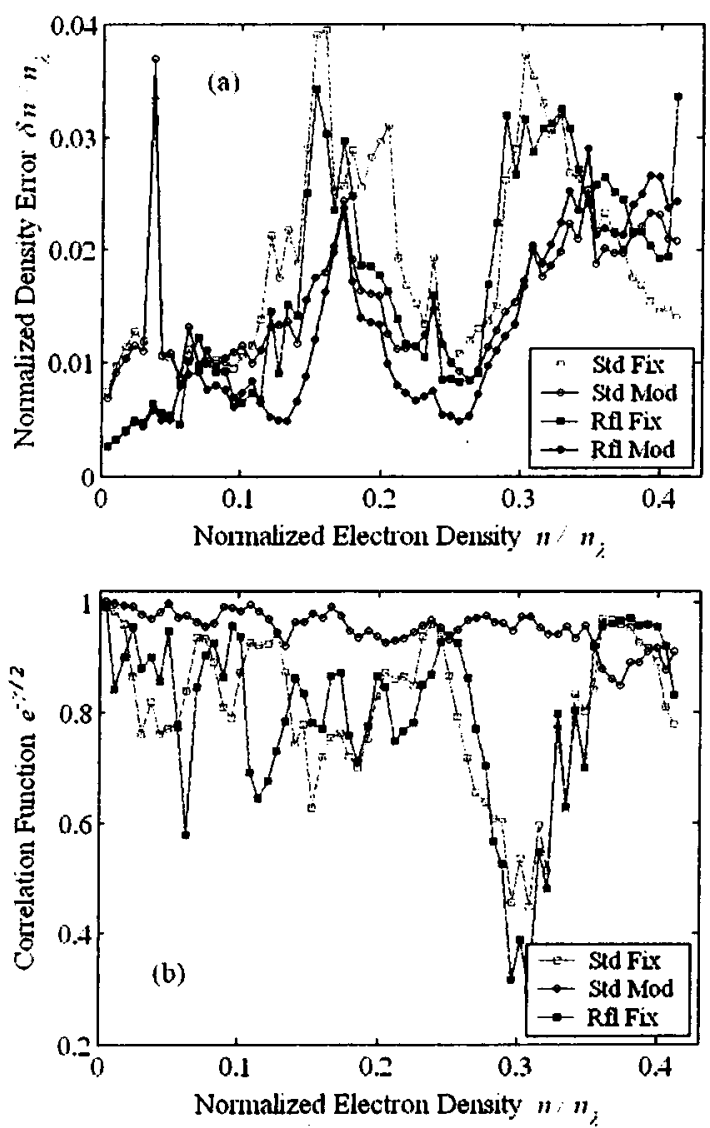

FIG 9 Distributions for the combined frequency (70/110 $\mathrm{GHz}$ ) VASIMR electron density measurements. (a) The normalized error bar $\delta n / n_{\lambda(70 \mathrm{GHz})}$, where $\delta n$ is the unit width. (b) The integral of the product of error populations, Eq. (17), for comparison with the reference model. The squares are fixed amplitude, the circles modulated amplitude. Open symbols are the standard model, solid symbols include reflection.

Table 4 IV Mean values, in percent, for the fractional error bars and differences for the VASIMR density data at 70 and $110 \mathrm{GHz}$, from the data of Fig. 9(a).

\begin{tabular}{|c|c|c|c|c|}
\hline & \multicolumn{2}{|c|}{ Standard $(\rho=0)$} & \multicolumn{2}{c|}{ Reflections $(\rho>0)$} \\
\cline { 2 - 5 } & $\begin{array}{c}\text { Fixed } \\
\alpha\end{array}$ & $\begin{array}{c}\text { Modulated } \\
\alpha\end{array}$ & $\begin{array}{c}\text { Fixed } \\
\alpha\end{array}$ & $\begin{array}{c}\text { Modulated } \\
\alpha\end{array}$ \\
\hline $\begin{array}{c}\text { combined } \\
\text { frequency } \\
\text { error bar }\end{array}$ & 19.6 & 14.4 & 10.2 & 7.54 \\
\hline $\begin{array}{c}\text { difference } \\
\text { between } \\
\text { frequencies }\end{array}$ & -0.076 & -2.11 & -3.49 & -4.92 \\
\hline $\begin{array}{c}\text { magnitude } \\
\text { of } \\
\text { difference }\end{array}$ & 22.9 & 16.2 & 17.9 & 14.3 \\
\hline
\end{tabular}

and the calibration data, then, the data reduction proceeds just as with the previous case. The total database is comprised of measurements made over a period of several months, and only measurements made at transverse positions $>5 \mathrm{~cm}$ from the viewport center were excluded. At $5 \mathrm{~cm}$, which is the nominal edge of the window, the scene beam signals are reduced by clipping typically to $10-15 \%$ of their central values, as determined from the calibration scans. The total number of phase shift measurements in the IEC database is 210. The measurements are monochromatic but data at two frequencies, 70 and $90 \mathrm{GHz}$, were used in separate test series. The quadrature shift was $54^{\circ}$ for the $90 \mathrm{GHz}$ data and varied from 24 to $39^{\circ}$ for the $70 \mathrm{GHz}$ data. The calibration resolution was typically about $0.0034 \mathrm{~cm}\left(3^{\circ}\right.$ at $70 \mathrm{GHz}$ ), higher than for the high-density data, and these scans were fit to the separate reflections model (Table I).

Histograms of this data are presented in Fig. 10. Most of the shifts are less than $2^{\circ}$, and all are less than $6^{\circ}$. In this case the difference distributions were compiled using the average of the results for the four models as the reference phase. The distribution means
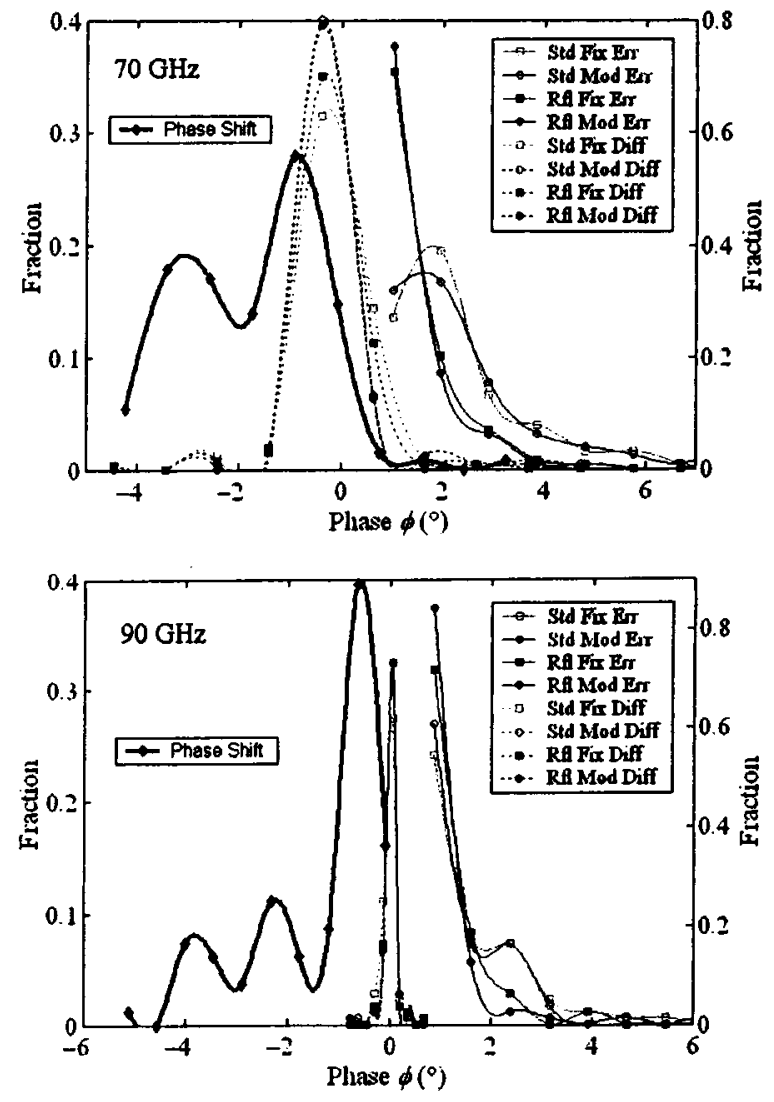

Figure 10 Distributions of phase shift measurements $\Delta \phi$ (heavy line, left axis), phase shift differences $\Delta \phi-\Delta \phi_{\text {ave }}$ (broken lines, right axis), and error bars $\delta \Delta \phi$ (solid lines, right axis), for the IEC data, taken in Ar and D2. 
Table V. Mean differences and errors, in degrees, for the IEC phase shift data at 70 and $90 \mathrm{GHz}$.

\begin{tabular}{|c|c|c|c|c|c|}
\hline & & \multicolumn{2}{|c|}{ Standard $(\rho=0)$} & \multicolumn{2}{c|}{ Reflections $(\rho>0)$} \\
\cline { 3 - 6 } & & $\begin{array}{c}\text { Fixed } \\
\alpha\end{array}$ & $\begin{array}{c}\text { Modulated } \\
\alpha\end{array}$ & $\begin{array}{c}\text { Fixed } \\
\alpha\end{array}$ & $\begin{array}{c}\text { Modulated } \\
\alpha\end{array}$ \\
\hline \multirow{2}{70}{$\begin{array}{c}\text { GHz } \\
\mathrm{GHz}\end{array}$} & $\begin{array}{c}\text { magnitude } \\
\text { of } \\
\text { difference }\end{array}$ & 0.599 & 0.556 & 0.564 & 0.553 \\
\cline { 2 - 6 } & error bar & 2.65 & 2.57 & 1.42 & 1.35 \\
\hline \multirow{2}{90}{$\begin{array}{c}\text { GHz } \\
\mathrm{GHz}\end{array}$} & $\begin{array}{c}\text { magnitude } \\
\text { of } \\
\text { difference }\end{array}$ & 0.0988 & 0.0931 & 0.0890 & 0.0902 \\
\cline { 2 - 6 } & error bar & 1.71 & 1.52 & 1.26 & 1.03 \\
\hline
\end{tabular}

are given in Table $\mathrm{V}$. It is seen that the difference distributions show little variation, and that the error bars are fairly insensitive to amplitude modulation, which was very small in this data. The mean values of the reflection coefficient were 0.032 and 0.019 , respectively, for 70 and $90 \mathrm{GHz}$. The fractional change in the calculated error bar with reflection terms remains significant, but the absolute size of the error is small in this data, less than $3^{\circ}$ for all cases.

\section{Conclusion}

The cable coupling of the transmitter and receiver in this instrument permits flexibility in the experimental configuration and, particularly, simple translational phase calibration. The translational calibration, in turn, permits modeling of residual reflections which occur between the transmitter and receiver and results in reduction of the measurement uncertainty. The quadrature configuration, in addition to eliminating sensitivity nulls and resolving phase change direction ambiguities at extrema, is critical in determining (monochromatic) phase shifts in the presence of significant amplitude modulation, which is typical in high-density data. The polychromaticity of the interferometer has proved useful here in error analysis for electron density measurements, by permitting combination of error distributions for simultaneous measurements at two frequencies, and in providing independent support for the analysis, from comparison of the simultaneous measurements themselves.

In the VASIMR experiment, temporally resolved interferometer data from plasma shots were analyzed to determine phase shifts and error bars using the standard phase model and a modified version including reflection terms. For both models, the data were also processed neglecting amplitude modulation by the plasma, for comparison. The measured phase shifts are from 0 to $530^{\circ}$, and the differences in phase shift among the models are typically 5 to $20^{\circ}$, with larger differences occurring for strong attenuation off quadrature. The phase shift error bars, calculated from a
Monte Carlo algorithm, show a weak phase dependence and have means from 3 to $15^{\circ}$; in the full model the mean errors are $6.8^{\circ}$ and $3.0^{\circ}$, respectively, for the two frequencies, 70 and $110 \mathrm{GHz}$. The inclusion of reflections reduced the average phase shift errors by a factor of 1.8 and the inclusion of modulation by a factor of 1.3. The error bars for the low frequency are larger than those for the high frequency by approximately a factor of 2, which compares to the (asymptotic) ratio 1.6 for the phase shifts themselves. Electron densities were calculated from the phase shifts at the two frequencies, and the difference in density between the two frequencies has mean magnitudes of $23 \%$ for the standard phase model and $14 \%$ for the full model. Combining the two frequency measurements in the uncertainty analysis results in mean density error bars of $20 \%$ and $7.5 \%$, for these respective models, with a general dependence on the model similar to that of the phase shifts.

In addition to the high-density work, a similar phase analysis of a collection of 70 and $90 \mathrm{GHz}$ data from the steady-state IEC device was performed. These data show very small phase shifts $\left(<2^{\circ}\right.$ typical) and negligible attenuation, with less variation in results among the models. The mean error bars for the full reduction were 1.4 and $1.0^{\circ}$ for the 70 and $90 \mathrm{GHz}$, respectively.

\section{Acknowledgment}

The authors wish to thank Franklin R. ChangDíaz and the VASIMR team at the Advanced Space Propulsion Laboratory, Johnson Space Center, NASA for providing the laboratory plasma and test facility for a portion of the data used in this investigation.

[1] M. Gilmore, W. Gekelman, K. Reiling, and W. A. Peebles, "A reliable millimeter-wave quadrature interferometer," http://128.97.43.7/lapd/Genera//Diagnostics/Interferometer/Int erferometer.html (June 27, 2003).

[2] Michael Friedrichs, http://www.fmt.uniwuppertal.de/English/research/diagnostic/MWI.html (March 11, 2002).

[3] M. A. Heald and C. B. Wharton, Plasma Diagnostics with Microwaves, John Wiley \& Sons, Inc., New York (1965).

[4] C. J. Buchenauer and A. R. Jacobson, "Quadrature interferometer for plasma density measurements," Rev. Sci. Instrum., Vol. 48, No. 7 (July 1977).

[5] A. P. Thorne, Spectrophysics, Chapman and Hall \& Science Paperbacks, London (1974).

[6] J.P Squire, F.R. Chang-Díaz, V.T. Jacobson, T.W. Glover, F.W. Baity, R.H. Goulding, R. Bentson, E.A. Bering III, K.A. 
Stokke, "Experimental Research Progress Toward the VASIMR Engine", $28^{\text {th }}$ International Electric Propulsion Conference, March 17-21, 2003, Toulouse, France.

[7] G. H. Miley, Y. Gu, J. M. DeMora, R. A. Stubbers, T. A. Hochberg, J. H. Nadler and R. A. Anderl, "Discharge Chararcteristics of the Spherical Inertial Electrostatic Confinement (IEC) Device," IEEE Transactions on Plasma Science 25 (4), pp. 733-739 (August, 1997).

[8] D. Veron, "Submillimeter Interferometry of High-Density Plasmas," in Infrared and Millimeter Waves, Vol. 2, Instrumentation (K. Button Ed.), Academic Press, Inc. (1979).

[9] H. W. Coleman and W. G. Steele, Jr., Experimentation and Uncertainty Analysis for Engineers, John Wiley and Sons, Inc. (1999). 